\title{
Zero-trans fats by enzymatic interesterification of blends beef tallow / rice bran oil
}

\author{
Nadia Segura* and Iván Jachmanián \\ Área de Grasas y Aceites, Facultad de Química, UdelaR, Gral Flores 2124, CP 11800, Montevideo, Uruguay
}

Received 12 August 2019 - Accepted 24 November 2019

\begin{abstract}
The aim of this study was to analyze in detail the changes produced by the enzymatic interesterification of BT/RBO blends (beef tallow/rice bran oil) at different proportions, as an alternative for production of trans free fats. It was observed that the increase in the oil content produced a range of the content of saturated fatty acids from 20 to $38 \%$ in the blend and a range of polyunsaturated fatty acids from 35 to $18 \%$. In TAG composition, the SUU and SSS type (S: saturated fatty acid; U: unsaturated fatty acid) increased in concentration as a result of interesterification process and this effect was more noticeable when the blend was richer in oil, arriving at 19 and $4 \%$ respectively in 50:50 BT/RBO blend. These variations in composition greatly improved the plastic range of BT. The process studied produced new trans free fatty materials with improving suitability as food ingredients. Therefore, promising new materials were developed.
\end{abstract}

Keywords: enzymatic interesterification / beef tallow / rice bran oil / trans fatty acid / Lipozyme TL IM

Résumé - Graisse zéro-trans par interestérification enzymatique de mélanges de suif de bœuf et d'huile de son de riz. Le but de cette étude était danalyser en détail les changements produits par linterestérification enzymatique de mélanges de suif de bœuf / huile de son de riz dans des proportions différentes, en tant qualternative à la production de graisses trans. Il a été observé que laugmentation de la teneur en huile produit des teneurs en acides gras saturés allant de 20 à $38 \%$ dans le mélange et en acides gras polyinsaturés allant de 35 à $18 \%$. En termes de composition des triglycérides, la concentration des types SUU et SSS (S : acide gras saturé ; U : acide gras insaturé) saccrot à la suite du processus dinterestérification et cet effet a été plus marqué lorsque le mélange était plus riche en huile, atteignant respectivement 19 et $4 \%$ dans un mélange 50:50 de suif de bœuf/huile de son de riz. Ces variations de composition ont grandement amélioré la plasticité du suif de bœuf. Le procédé étudié a permis de produire de nouvelles matières grasses zéro-trans plus adaptées en tant quingrédients alimentaires. Par conséquent, de nouveaux matériaux prometteurs ont été développés.

Mots clés : interestérification enzymatique / suif de bœuf / lhuile de son de riz / acide gras trans / lipozyme TL IM

\section{Introduction}

The adverse health effect of trans fatty acids (TFA) intake and in particular its direct relationship to the risk of cardiovascular disease is well known. Along these lines, in recent years, more countries have begun to apply regulations focused on reducing the consumption of industrial TFA. In 2015 , the FDA declared partially hydrogenated fats as nonGRAS, giving to industry three years for its elimination of processed foods. In Uruguay, the regulation dates from 2018, reaching industrial TFA to a maximum of $2 \%$ in direct

$\overline{\text { *Correspondence: nsegura } @ f q . e d u . u y ~}$ consumption fats (oils, fats and margarines) and $5 \%$ in the fat of the rest of the food from November 2019. As of May 2022, the maximum limit will be $2 \%$ in fats from food for direct or industrial use. The regulation excludes trans fatty acids from ruminates.

For these reasons, the search for alternatives for the substitution of TFA in products such as shortenings and margarines, traditionally produced by partial hydrogenation of vegetable oils, has been a topic of great interest to the different actors involved in the field of edible fats. Among the different alternatives, interesterification of fatty materials of different origin appears as one of the most attractive given its versatility to design products of suitable plasticity and physicochemical properties. 
Although chemically catalyzed interesterification has been traditionally used by industry and well known industrial procedures and equipment are readily available, lipase catalysis has become an interesting alternative, since reactions are more specific, it requires milder reaction conditions and less waste can be produced.

There are a few records about the use of rice bran oil (RBO) as an important ingredient in the formulation of shortenings of different types, suggesting its blend with oils of a different nature and having the blend go through interesterification processes. For example, its chemical interesterification with palm oil and palm stearin on a pilot scale has been studied in different proportions to obtain products with suitable melting profiles for a multi purpose shortening, optimizing the crystallization variables to get a fat phase useful for making margarine (Mayamol et al., 2009). Also, enzymatic catalysis (with Lipozyme TL IM lipase) has been studied for similar applications, demonstrating that due to the high content of palmitic acid from palm oil, it was possible to obtain $\beta$ crystals mainly (Reshma et al., 2008).

One of the particular characteristics of RBO is the high content of unsaponifiable lipid compounds, which can range from 3 to $5 \%$ depending on the rice variety and the method used for the extraction (Ghosh, 2007; Goffman et al., 2003; Van Hoed et al., 2006). This unsaponifiable fraction contains a complex mixture of compounds with antioxidant properties giving the oil a very high resistance to oxidation ( $\mathrm{Xu}$ and Godber, 1999). Among these compounds, there are several tocopherols, tocotrienols and oryzanols, the latter being the most characteristic of this oil. The antioxidant properties exhibited by oryzanols are associated with the structure of ferulic acid, a potent antioxidant. It has been shown that because of these properties, the intake of RBO is beneficial for human health, given their efficient antimutagenic and anticarcinogenic effects (Xu and Godber, 1999). Depending on the characteristics of the refining process used, some of these bioactive compounds are lost, but some of them are preserved in the refined oil, so any food product containing RBO will benefit from their properties thereof (Van Hoed et al., 2006).

Beef tallow (BT) is an abundant fat raw material and for various reasons interesting for the food industry. It is produced as a by-product of the meat industry, which is one of the most important in Uruguay. In addition, other advantages of this fatty material as an ingredient in foodstuff are its high thermal and oxidative stability, its adequate plasticity at temperatures slightly above room temperature and its typical taste, much appreciated by customers used (Grompone and Moyna, 1983; Jachmanián et al., 2002).

In spite of this, beef tallow presents physicochemical characteristics that make it inconvenient for many food applications. Among them, the high solid fat content in the temperature range between 32 and $38^{\circ} \mathrm{C}$ stands out, this produces an undesirable residual waxy" mouthfeel. In addition, it has a significant content of trisaturated triglycerides ( $\sim 13 \%$ ) which contribute to the formation of large crystals (diameters of 2 to $3 \mathrm{~mm}$ or larger), resulting in a hard product with an undesirable sandy" mouthfeel (Jin et al., 2007).

Another concern is related to its nutritional properties, mainly due to its high content of saturated fatty acids $(\sim 57 \%)$, and, to a lesser extent, to the presence of cholesterol $(\sim 0.12 \%)$
(Grompone and Moyna, 1983). On the other hand, it has a contribution of TFA ( $\sim 5$ to $6 \%$ ), in particular trans vacenic acid and CLA, that are beneficial for health (DAgostini and Mancini, 2012).

The aim of this study was to analyze in detail the changes produced by the enzymatic interesterification of blends BT/RBO at different proportions, as an alternative for manufacturing trans free fats. The effect of the proportion of $\mathrm{RBO}$ added to BT on triglycerides (TAG) composition and melting thermograms were evaluated.

\section{Experimental procedures}

\subsection{Materials}

First jus beef tallow was kindly provided by Frigorífico Tacuaremb S.A., Tacuaremb, Uruguay. Refined rice bran oil (ARROZUR, Treinta y Tres, Uruguay) was acquired in the local market.

Lipase form porcine pancreas was supplied by SigmaAldrich (PPL Type II, activity equal to $11.6 \mathrm{U} / \mathrm{mg}$, in $\mu \mathrm{mol}$ of fatty acids hydrolyzed per minute per $\mathrm{mg}$ lipase, not immobilized lipase). Lipozyme TL IM (from Themomices lanuginosus, immobilized lipase) was gently provided by Novozymes, Denmark.

Organic solvents, analytical standards and reagents used were supplied by Dexin S.R.L, Montevideo, Uruguay (agent for Sigma-Aldrich Company).

\subsection{Interesterification reactions}

Blends with different proportions of $\mathrm{BT}$ and $\mathrm{RBO}$ were prepared (containing 10, 20, 30, 40 and $50 \mathrm{wt} . \%$ of RBO). Blends were heated at $60^{\circ} \mathrm{C}$ and magnetically stirred for 15 min until total homogenization. Seven grams of each blend were transferred to screw cap tubes and $0.7 \mathrm{~g}$ of Lipozyme TL IM was added. The tubes were vented with $\mathrm{N}_{2}$ and placed inside an orbital shaker at $60^{\circ} \mathrm{C}, 200 \mathrm{rpm}$, for $24 \mathrm{~h}$. After the incubation period, the enzyme was separated from products by centrifugation (3000 rpm, $15 \mathrm{~min}$ ) and the lipid fraction then tested.

\subsection{Fatty acid composition}

Pure raw materials ( $\mathrm{RBO}$ and $\mathrm{BT}$ ) were treated with $\mathrm{BF}_{3} /$ $\mathrm{MeOH}$ according to AOCS Ce $1 \mathrm{~b}-89$ in order to transform the TAG to the corresponding methyl esters. The esters were analyzed by capillary gas chromatography (GC), using a Shimadzu GC-2014 equipped with FID and a capillary column SP $2330(25 \mathrm{~m} \times 0.5 \mathrm{~mm} \times 0.25 \mu \mathrm{m})$. The temperature program started at $160{ }^{\circ} \mathrm{C}$, followed by a heating step $\left(4^{\circ} \mathrm{C} / \mathrm{min}\right)$ until $230^{\circ} \mathrm{C}$, and then held at $230^{\circ} \mathrm{C}$ for $10 \mathrm{~min}$. Nitrogen at $40 \mathrm{kPa}$ was used as carrier gas, with a split ratio of 1:80. Fatty acids were then identified and quantified. Fatty acid composition of the different blends were calculated considering the composition of both pure raw materials and their proportion in the blend. Fatty acid composition of products were considered identical to that of the corresponding blend, considering that interesterification does not modify this parameter. 
Table 1. Fatty acid composition of pure BT and RBO and their blends in different proportions.

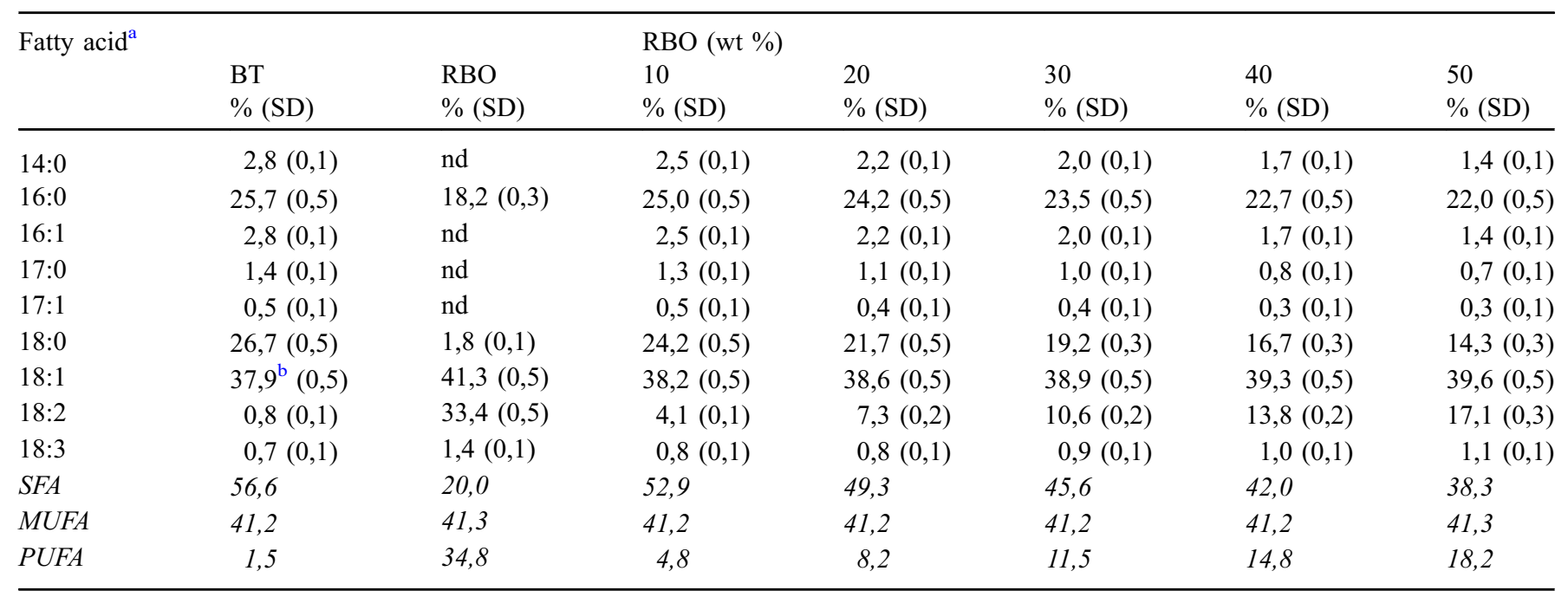

SFA: saturated fatty acids; MUFA: monounsaturated fatty acids; PUFA: polyunsaturated fatty acids; nd: not detected; SD: standard deviation. Values in italics indicate the sum of FA classes.

${ }^{\text {a }}$ Only fatty acids above $0.5 \%$ in BT or RBO were selected and shown.

${ }^{\mathrm{b}}$ It includes approximately $5 \%$ of the trans isomer.

Analyses were performed in duplicate and average results were reported.

\subsection{Triacylglycerol composition}

The TAG composition of both raw materials and products were determined by reverse phase high performance liquid chromatography (HPLC) based on the equivalent carbon number (ECN), defined as $\mathrm{CN}-2 \mathrm{n}$ " (where $\mathrm{CN}$ is the number of carbons in the TAG, excluding the three in the glycerol backbone, and $n$ is the number of double bonds). Analyses were carried out using an HPLC Shimadzu Prominence 20A (Shimadzu, Corporation, Kyoto, Japan), equipped with an evaporative light scattering detector Shimadzu ELSD-LTII and two columns Supelcosil TM C18 $(25 \mathrm{~cm} \times 4.6 \mathrm{~mm} \times 5 \mu \mathrm{m})$. The test started with a flow rate of $1 \mathrm{~mL} / \mathrm{min}$ of an acetone/ acetonitrile (1:1) mixture, with an increasing linear gradient of chloroform up to $20 \%$ after $60 \mathrm{~min}$, solvent composition was then held for $20 \mathrm{~min}$ and finally returned to the initial composition after $85 \mathrm{~min}$. Peaks were identified using pure TAG standards and considering the order of elution according to the corresponding ECN.

TAG composition of the different blends were calculated considering the composition of both pure raw materials and their proportion in the blend, this procedure was found to be simpler and more accurate that the analysis of the final blend.

Analyses were performed in duplicate and average results were reported.

In order to easily analyze the changes produced by the interesterification process, TAG were grouped by type and the percentage of Relative variation" (RV) of the different TAG types were calculated as:

$$
R V=100 \frac{\left(T A G_{i}\right)_{P}-\left(T A G_{i}\right)_{B}}{\left(T A G_{i}\right)_{B}},
$$

$R V$ : percentage of relative variation; $\left(\mathrm{TAG}_{\mathrm{i}}\right)_{\mathrm{P}}$ : concentration of TAG type i" in the product; $\left(\mathrm{TAG}_{\mathrm{i}}\right)_{\mathrm{B}}$ : concentration of TAG type i" in the original blend.

\subsection{Analysis of fatty acids at the sn-2 position}

The nature of fatty acids at the $s n-2$ position of triglycerides was determined according to the AOCS method Ch 3-91. This method consists of partial hydrolysis of the TAG by pancreatic lipase during a determined time, separation of the 2- monogycerides by TLC, and derivatization to methyl esters and there analyze by GC.

\subsection{DSC analysis}

Thermal profiles and solid fat content (SC) curves were determined by differential scanning calorimetry (DSC), using a calorimeter TA Q20 (TA Instruments), equipped with a Refrigerated Cooling System RCS90, according to the AOCS method $\mathrm{Cj}$ 1-94. The peak areas, the partial areas and the percentage of SC were determined from the melting profiles using the software TA Universal Analysis 2000 (version 3.9A). Calibration of the DSC equipment was performed using metallic Indium as standard.

\section{Results and discussion}

\subsection{Fatty acid composition}

The fatty acid composition of BT and RBO and their blends in different proportions is shown in Table 1. It was observed that the increase in the oil content produced a gradual decrease in saturated fatty acids in the blend and increased polyunsaturated fatty acids. 
Table 2. Triacylglycerols composition of pure BT and RBO, their blends in different proportions and the enzymatic interesterification products.

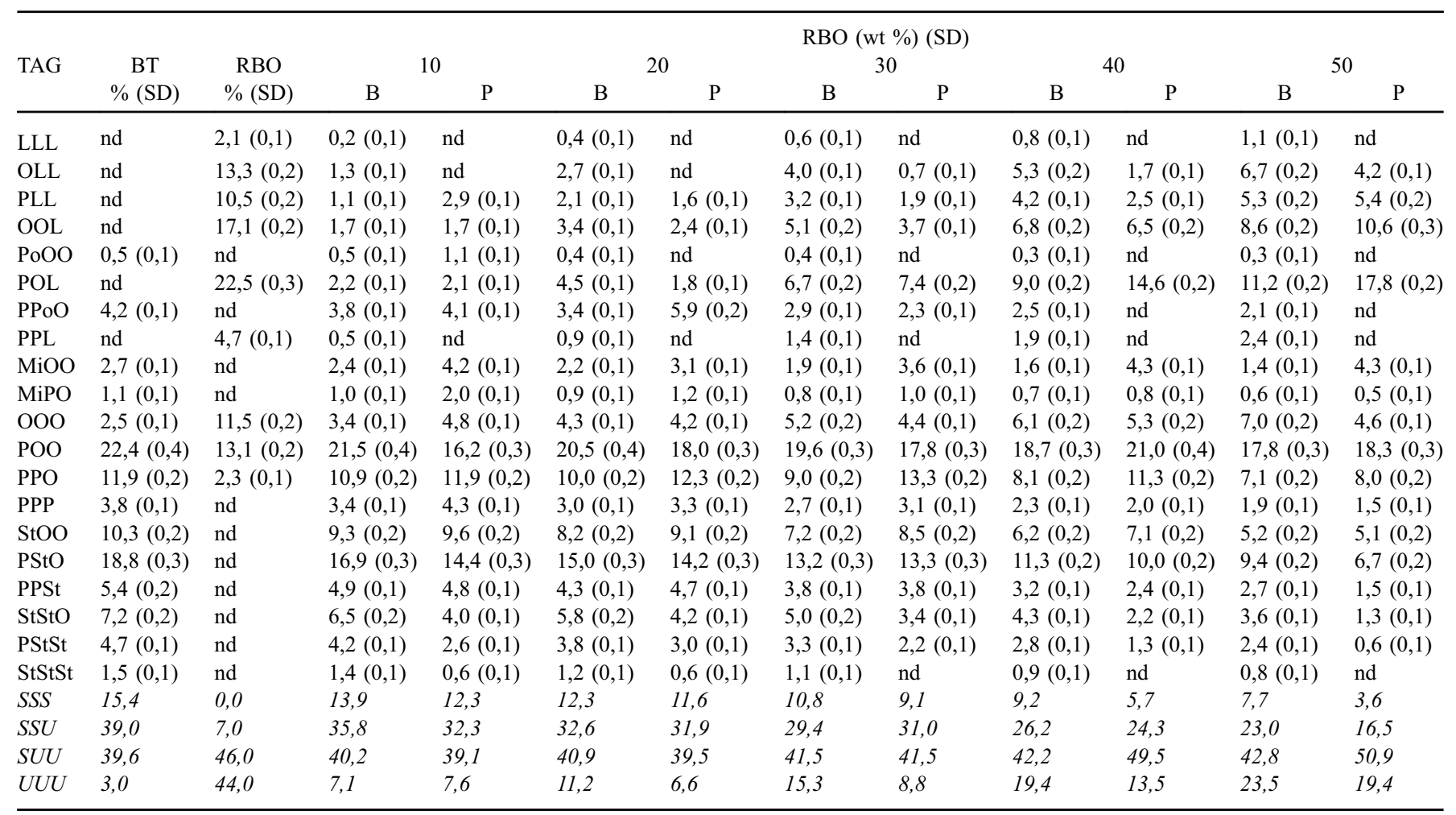

B: blend; P: interesterification product (24 h); Mi: myristic (14: 0); P: palmitic (16: 0); Po: palmitoleic (16: 1); St: stearic (18: 0); O: oleic (18: 1); L: linoleic (18: 2); SSS: trisaturated; SSU: monounsaturated disaturated; SUU: diunsaturated monosaturated; UUU: triunsaturated. nd: not detected; SD: standard deviation.

The composition of both pure fatty materials are in accordance with the compositions established by the Codex Alimentarius. The composition of BT falls within the range of the study conducted by Grompone and Moyna (1983) on BT of Uruguayan origin, instead it shows a slight difference with the composition shown by Lo and Handel (1983), these authors found that BT had a higher content of oleic acid $(45.9 \%)$ and linoleic acid (1.7\%) and a lower content of stearic acid (20.2\%) than were observed in this work (37.9, 0.8 and $26.7 \%$ respectively). Regarding RBO compared to the study conducted by Reshma et al. (2008), a lower palmitic content (18.2\%) and higher linoleic content (33.4\%) was found than that reported by these authors (28.1 and $28.3 \%$ respectively).

\subsection{Triacylglycerols composition}

Table 2 shows the TAG composition of BT and RBO, their blends in different proportions and the products obtained by enzymatic interesterification. As mentioned above, TAG composition of the blends was obtained by calculation from the composition of the pure materials.

Most TAG constituents of by RBO correspond to the UUU and SUU types (44.0 and $46.0 \%$, respectively). While BT showed its TAG population distributed mainly between the two intermediate saturation degree groups: SSU and SUU, with 39.0 and $39.6 \%$ respectively. Therefore, in the blends, the most saturated TAG decreased and the UUU type increased as the oil content increased.

The variation of the RV values as a function of the percentage of RBO in the blends is shown in Figure 1.

TAG of the SUU type increased in concentration as a result of interesterification and this effect was more important as the proportion of oil in the blend increased. The main TAG of this group were POL from RBO, StOO from BT and OOP from both. This effect can be explained by incorporation of saturated fatty acid to UUU TAG type characteristic of the oil, a higher oil content as much UUU TAG type available of accepting a saturated fatty acid.

The concentration of TAG of the SSS type decreased as a result of interesterification and this effect was more noticeable as the blend got richer in oil. The contribution of this group of TAG was exclusive from BT, reason why this effect was expected. The same was also observed in previous studies with high oleic sunflower oil (HOSFO) (Segura et al., 2011a).

Concentration of TAG type UUU, coming exclusively from RBO, and of type SSU, coming mainly from BT, showed a decrease by the effect of interesterification, but the effect of the dilution with oil was unclear.

Observing in a global way, its notorious that by increasing amount of oil in the blend, there is a higher content of 
Table 3. Percentage of each fatty acid occupying the sn-2 position of BT/RBO blends in different proportions and their enzymatic interesterification products.

\begin{tabular}{|c|c|c|c|c|c|c|c|c|c|c|}
\hline \multirow[t]{3}{*}{ FA } & \multicolumn{10}{|c|}{ RBO (wt \%) (SD) } \\
\hline & \multicolumn{2}{|c|}{10} & \multicolumn{2}{|c|}{20} & \multicolumn{2}{|c|}{30} & \multicolumn{2}{|c|}{40} & \multicolumn{2}{|c|}{50} \\
\hline & $\mathrm{B}$ & $\mathrm{P}$ & $\mathrm{B}$ & $\mathrm{P}$ & B & $\mathrm{P}$ & B & $\mathrm{P}$ & $\mathrm{B}$ & $\mathrm{P}$ \\
\hline $16: 0$ & $16,8(0,8)$ & $35,1(1,8)$ & $15,8(0,8)$ & $37,1(1,9)$ & $14,8(0,7)$ & $35,3(1,8)$ & $13,7(0,7)$ & $38,3(1,9)$ & $12,5(0,6)$ & $38,0(1,9)$ \\
\hline $16: 1$ & $48,8(2,4)$ & $33,9(1,7)$ & $48,8(2,4)$ & $38,7(1,9)$ & $48,8(2,4)$ & $35,0(1,8)$ & $48,8(2,4)$ & $37,2(1,9)$ & $48,8(2,4)$ & $40,3(2,0)$ \\
\hline $17: 0$ & $23,8(1,2)$ & $34,1(1,7)$ & $23,8(1,2)$ & $38,0(1,9)$ & $23,8(1,2)$ & $35,9(1,8)$ & $23,8(1,2)$ & $38,7(1,9)$ & $23,8(1,2)$ & $40,6(2,0)$ \\
\hline $18: 0$ & $17,2(0,9)$ & $31,8(1,6)$ & $17,1(0,9)$ & $34,2(1,7)$ & $17,0(0,9)$ & $33,6(1,7)$ & $16,9(0,8)$ & $34,6(1,7)$ & $16,7(0,8)$ & $36,7(1,8)$ \\
\hline $18: 1$ & $42,8(2,1)$ & $30,9(1,5)$ & $42,1(2,1)$ & $31,5(1,6)$ & $41,5(2,1)$ & $32,5(1,6)$ & $40,8(2,0)$ & $33,6(1,7)$ & $40,2(2,0)$ & $35,4(1,8)$ \\
\hline $18: 2$ & $52,2(2,6)$ & $11,2(0,6)$ & $51,1(2,6)$ & $13,1(0,7)$ & $50,7(2,5)$ & $21,7(1,1)$ & $50,4(2,5)$ & $14,8(0,7)$ & $50,3(2,5)$ & $18,1(0,9)$ \\
\hline $18: 3$ & $23,4(1,2)$ & nd & $19,0(1,0)$ & nd & $15,4(0,8)$ & $7,3(0,4)$ & $12,2(0,6)$ & $8,0(0,4)$ & $9,5(0,5)$ & $9,3(0,5)$ \\
\hline$S F A$ & 19,0 & 33,9 & 18,4 & 36,3 & 17,7 & 34,9 & 16,8 & 37,1 & 15,8 & 37,8 \\
\hline
\end{tabular}

B: blend; P: interesterification product $(24 \mathrm{~h})$; nd: not detected; SFA: saturated fatty acids; MUFA: monounsaturated fatty acids; PUFA: polyunsaturated fatty acids; SD: standard deviation. Values in italics indicate TAG classes.

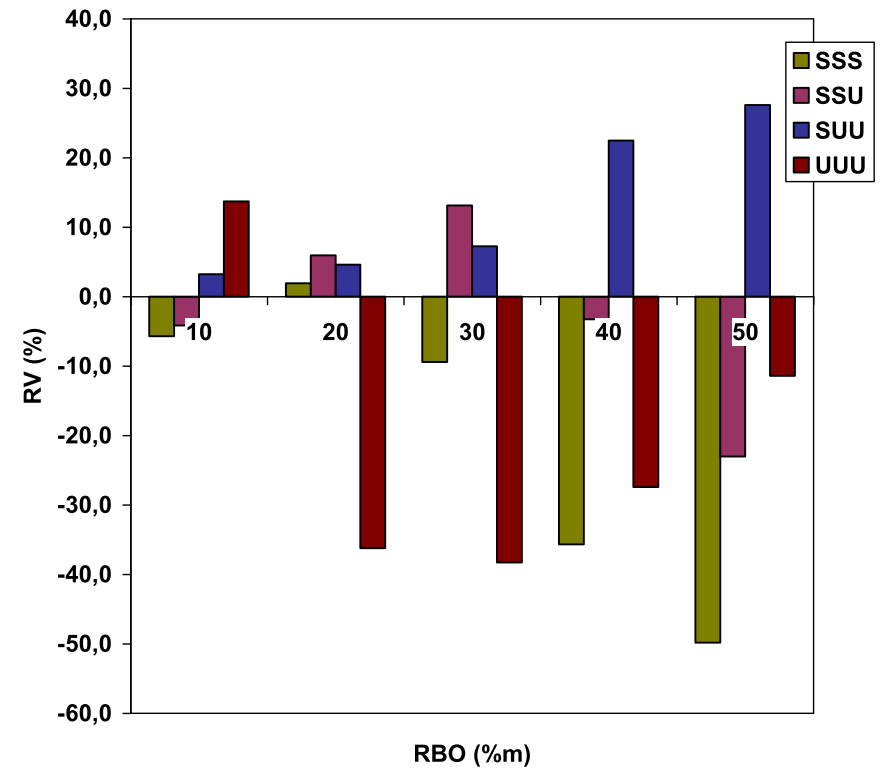

Fig. 1. Relative variation (RV) for each type of TAG produced by enzymatic interesterification of BT / RBO blends in different proportions.

unsaturated fatty acids and a lower number of saturated ones, which would favor a greater formation of TAG with a high amount of unsaturated ones (UUU and SUU), but in an interstrification with low or no specificity, homogenous TAG are not favored, which is in accordance with the results obtained.

Similar effect were observed by Criado et al. (2007) by intersterifying virgin olive oil with fully hidogenated fat, they obtain products enriched in high saturated TAG as they increase the fully hidogenated fat content in the blend and higher content of high unsaturated TAG when they increase the oil content in the blend. Imran and Nadeem (2015) also report that intersterification decreases the content of homogenous TAG and increases heterogens TAG by interstrifying canola oil and fully hydrogenated cotton oil.

TAG of SUU type is related to functional and sensory characteristics of the fatty material (OBrien, 2009), so, given the results of composition obtained, is expected that enzymatic intesterification products of BT/RBO blends find application in food industry.

\subsection{Fatty acid composition of the sn-2}

From the composition of fatty acids in the $s n-2$ position determined for each sample and taking into account the overall composition, the percentage distribution of each fatty acid between the $s n-2$ positions and the sum of the $s n-1$ and $s n-3$ positions was calculated (Table 3 ).

The products of the $\mathrm{BT} / \mathrm{RBO}$ blends showed a variation in the sn- 2 position, that did not reach $33 \%$ but got around it. Interesterification could therefore be considered to have occurred without positional specificity. This behavior had already been observed for the enzymatic interesterification of BT blends with high oleic sunflower oil (Segura et al., 2011a). This was attributed to the acylmigration of fatty acids. This process accelerates with increasing temperature and occurs to a greater extent in prolonged incubations such as the one carried out in this work (Xu et al., 1998).

\subsection{Melting thermograms}

Figure 2 shows the melting thermogram of the pure materials. The thermal behavior of BT and its interesterification product was already discussed in earlier work done by the authors (Segura et al., 2011a). 


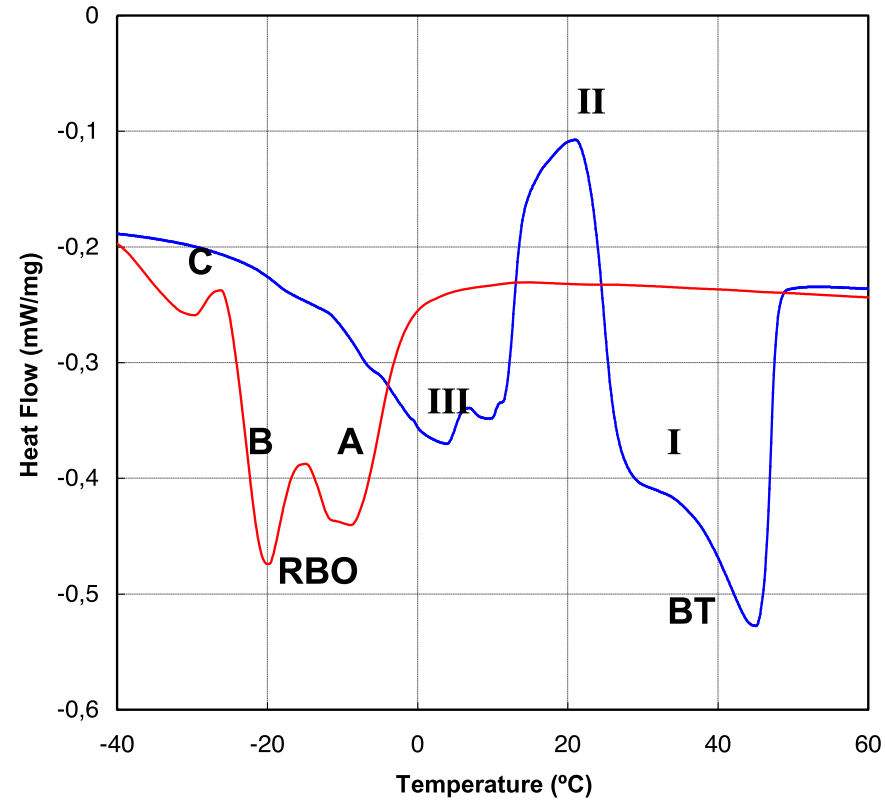

Fig. 2. Heating thermograms of Beef tallow (BT) and Rice bran oil (RBO).

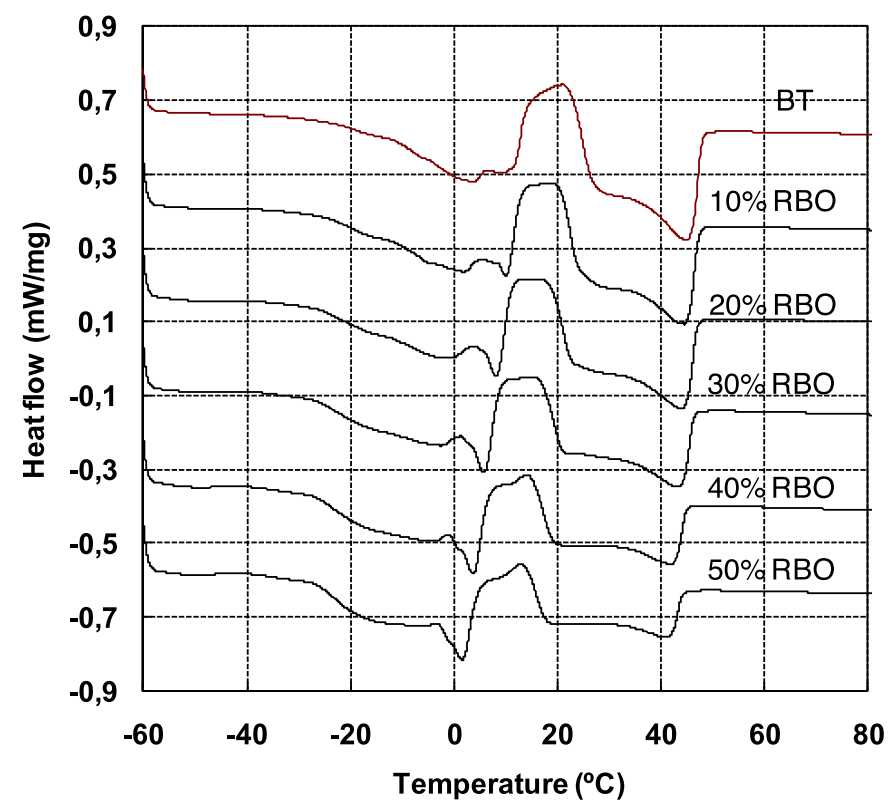

Fig. 3. Heating thermograms of $\mathrm{BT} / \mathrm{RBO}$ blends.

The RBO thermogram had at least three partially overlapping peaks, with the main two at temperatures of -19.8 (B) and $-8.9^{\circ} \mathrm{C}(\mathrm{A})$ and a smaller one at a lower temperature $(\mathrm{C}$; $\left.\mathrm{Tp}=-29.7^{\circ} \mathrm{C}\right)$. This overlap was expected considering the very diverse composition of this oil with no predominant TAG. Peak A could be considered as the melting of TAG of the SUU type (46\%), while the other two could correspond mainly to the melting of UUU-type TAG (44\%).

The blends of BT with RBO (Fig. 3) showed a decrease in the endothermic peak of higher temperature (peak 1) which

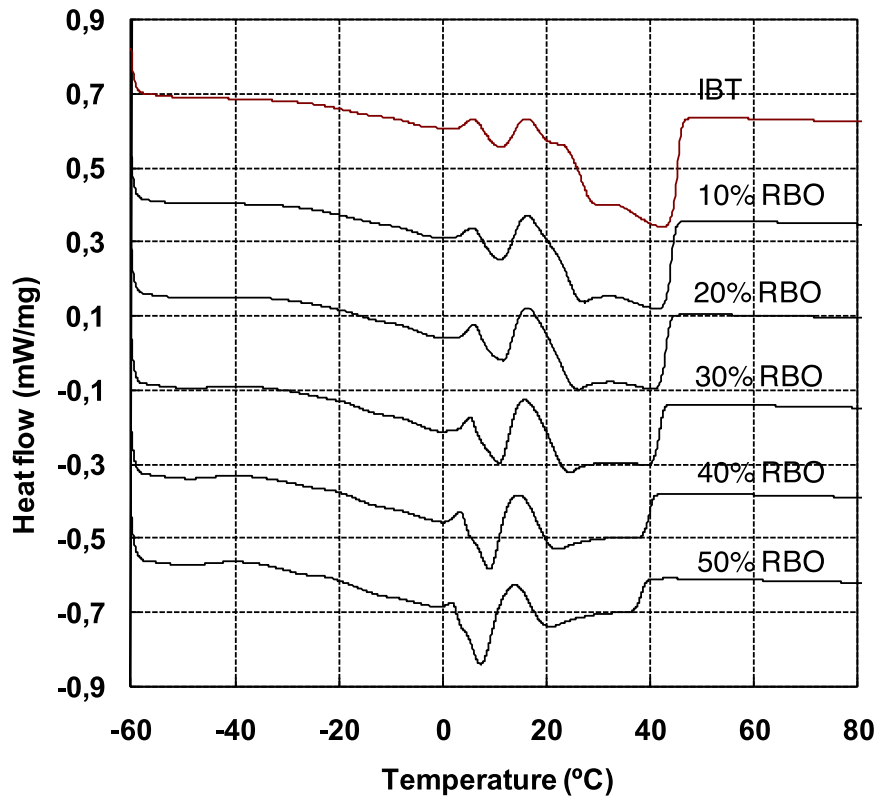

Fig. 4. Heating thermograms of interesterification products of BT and $\mathrm{BT} / \mathrm{RBO}$ blends.

became more pronounced as the proportion of oil in the blend increased. This was due to the dilution of the most saturated TAG of BT with the oil. The increase in the amount of oil also increased the area of the set of endothermic peaks at low temperature (peaks 3 and 4), which was an expected behavior since pure RBO showed peaks below $0{ }^{\circ} \mathrm{C}$ (Fig. 2).

A decrease in the size of the exothermic peak was also observed with the increase of oil in the mixture corresponding to the polymorphic transition $\beta \rightarrow \beta$ in $\mathrm{BT}$, which was consistent with the progressive dilution of BT. It was interesting to note that this peak completely disappeared after the interesterification of the mixtures (Fig. 4), a phenomenon that was also observed in the case of pure BT and blends of BT with HOSFO in previous experiences (Segura et al., 2011a). This could suggest that the interesterification products of these blends also showed a low tendency to the formation of $\beta$ crystals. This would be an advantage if these fats were to be used as a basis for margarines or shortenings, since $\beta$ crystals are smaller and softer than $\beta$ (OBrien, 2009).

For the interesterification products (Fig. 4), a decrease in the area of the endothermic peak at a higher temperature (peak 1) was observed after interesterification, as well as a shift to lower temperatures. This effect was accentuated by the increase in the amount of oil in the original mixture. Such behavior was expected since, as discussed in 3.2, the concentration of TAG with higher melting points (more saturated) decreased as a result of the process, which was accentuated by the increase in oil content. Similar effects were observed by Pang et al. (2019) by interesterifying a blend of BT, palm stearin and Camellia oil, these authors report the decrease and/or disappearance of peaks corresponding to high and low temperatures, increasing and/or appearing peaks at intermediate temperature, they relate this effect to the decrease in homogenous TAG and the increase in heterogeneous TAG due to intersterification. 
As for the second endothermic peak, peak 3 for the blends, after interesterification, it was split into two peaks (peaks 3 and 4 in the products). By increasing the amount of oil, peak 4 reduced its area until it became a shoulder of peak 3 , which in turn grew with the increase in oil content. This effect could be related to the increase in the inter-solubility of the TAG that composed the products, due to the effect of the observed randomization (OBrien, 2009).

\subsection{Solid fat content estimation from DSC thermograms (SC)}

By integrating the signal of the melting thermograms is possible estimate the variation of the solids content as a function of temperature. Although it is known that the results obtained by this methodology rarely coincide with those obtained for SFC by NMR (Timms, 2003), it allows an approximate analysis of the variation of solids content with temperature. Figure 5 shows the percentage of solids obtained by this method for blends and itneresterification products. It is observed that the distortion produced by the exothermic polymorphic transition observed in the melting thermograms of the blends distorts the SC curves of the blends by the appearance of an ascending section between 10 and $20^{\circ} \mathrm{C}$ (Fig. 5A). The increase in the amount of oil also generated a gradual decrease in the $\mathrm{SC}$ values at a given temperature, which was obviously linked to the dilution of the more saturated TAG of BT. This effect coincided with that previously reported for blends of BT with HOSFO (Segura et al., 2011a) and by other authors for blends with sunflower oil (Rodríguez et al., 2001) or with soybean oil (Lo and Handel, 1983).

Unlike the SC curves of the blends, those obtained for the products decreased continuously with the increase in temperature (Fig. 5B), not showing the distortion corresponding to the exothermic peak of the melting thermograms. At a given temperature, the interesterified material had a lower SC than its corresponding blend.

The plasticity of an edible fat product depends on the amount of solids and the variation of the solid fat content with the temperature. The amplitude of the melting range and other factors such as the crystalline morphology determine the range within which a fat can be considered plastic (Rao et al., 2001). According to De Man (1992), a fatty material can be considered to have adequate spreadability if the solids content is within the range of 15 to $35 \%$, which is called "plastic range".

As seen in Figure 5, the plastic range for the blends presented a wider temperature range as the oil content increased, in addition to a shift to lower temperatures. Interesterification process generated a shift of the plastic ranges to lower temperatures. This effect was also observed in previous studies with BT mixtures with HOSFO (Segura et al., 2011a), although for BT/HOSFO case, the displacement observed was greater. On the other hand, in the case of BT/ $\mathrm{RBO}$ blends, the interesterification process produced a slight decrease in the amplitude of the plastic range, as opposed to what was observed for BT/HOSFO blends.

It is interesting to note that the products obtained from blends containing at least $40 \%$ oil showed a plastic range at room temperature and a moderate amount of solids at $37^{\circ} \mathrm{C}$,

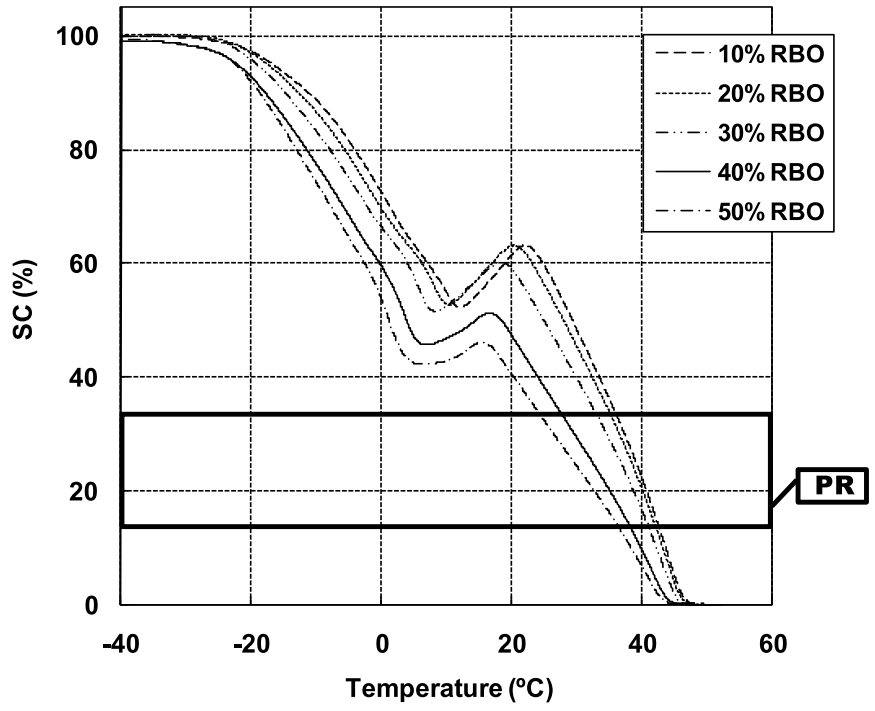

A)

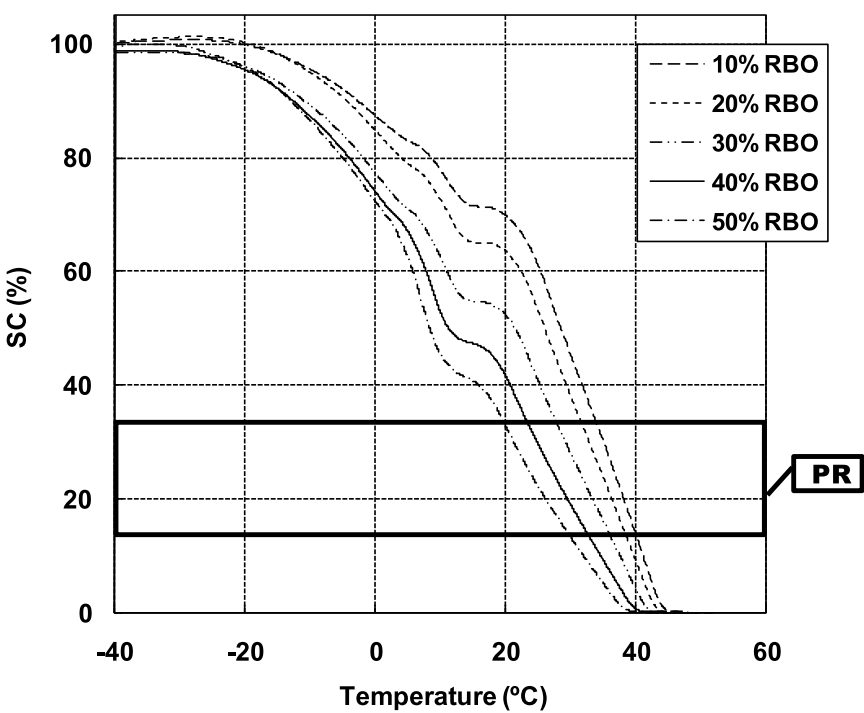

B)

Fig. 5. SC curves obtained by integration of DSC signal from the melting thermograms of RBO / BT blends (A) and their interesterification products $(\mathrm{B})$. PR: plastic range.

which makes them attractive fatty materials for manufacturing food products.

\section{Conclusion}

The enzymatic interesterification of $\mathrm{BT} / \mathrm{RBO}$ blends produced new trans free fatty materials. Their fatty acid composition changed accordingly with the proportion of each raw material in the original blend. The interesterification produced modifications in the TAG compositions of the blends which significantly affected the physicochemical properties of the end products improving their suitability as food ingredients. Therefore, promising new materials were developed. 
Acknowledgements. The authors thank PEDECIBA (Programa de Desarrollo de las Ciencias Básicas), CAP (Comisin Académica de Posgrado, Universidad de la República) and ANII (Agencia Nacional de Investigacin e Innovacin) for the financial support and scholarships.

\section{References}

Criado M, Hernández-Martín E, Lpez-Hernández A, Otero C. 2007. Enzymatic interesterification of extra virgin olive oil whit a fully hydrogenated fat. Characterization of the reaction and its products. J Am Oil Chem Soc 84: 717-726.

DAgostini D, Mancini Filho J. 2012. Ácidos grasos trans: características nutricionales. In Block JM, Barrera-Arellano D, eds. Temas selectos en aceites y grasas. Volumen 2-Química. Sao Paulo: Blucher, pp. 85-117.

De Man JM. 1992. Fats and oils: chemistry, physics and applications. In: Hui HD, ed. Encyclopedia of food science and technology. New York: John Wiley \& Sons, pp. 823-824.

Ghosh M. 2007. Review on recent trends in rice bran oil processing. J Am Oil Chem Soc 84: 315-324.

Goffman FD, Pinson S, Bergman C. 2003. Genetic diversity for lipid content and fatty acid Profile in rice bran. J Am Oil Chem Soc 80: 485-490.

Grompone MA, Moyna P. 1983. Characteristics of uruguayan beef tallow. J Am Oil Chem Soc 60: 1331-1332.

Imran M, Nadeem M. 2015. Triacylglycerol composition, physicochemical characteristics and oxidative stability of interesterified canola oil and fully hydrogenated cottonseed oil blends. Lipids Health Dis 14: 138.

Jachmanián I, Gil M, Grompone MA. 2002. Mejoramiento de las propiedades térmicas y nutricionales de la grasa vacuna uruguaya mediante interesterificacin enzimática. Aceites y Grasas 46: 84-92.

Jin Q, Gao H, Shan L, Liu Y, Wang X. 2007. Study on grainy crystals in edible beef tallow shortening. Food Res Int 40: 909-914.

Lo Y, Handel A. 1983. Physical and chemical properties of randomly interesterified blends of soybean and tallow for use as margarine oils. J Am Oil Chem Soc 60: 815-818.

Mayamol PN, Balachandran C, Samuel T, Sundaresan A, Arumughan C. 2009. Zero trans shortening using rice bran oil, palm oil and palm stearin through interesterification at pilot scale. Int $J$ Food Sci Technol 44: 18-28.

Official Methods and Recommended Practices of the American Oil Chemists Society. 1990. 4th ed. Walker, R. E., Ed. American Oil Chemists Society: Champaign, IL.

OBrien RD. 2009. Fats and oils. Formulating and processing for applications. Boca Raton: CRC Press.

Pang M, Ge Y, Cao L, Cheng J, Jiang S. 2019. Physicochemical properties, crystallization behavior and oxidative stabilities of enzymatic interesterified fats of beef tallow, palm stearin and camellia oil blends. J Oleo Sci. DOI: 10.5650/jos.ess 18201.

Rao R, Sankar KU, Sambaiah K, Lokesh BR. 2001. Differential scanning calorimetric studies on structured lipids from coconut oil triglycerides containing stearic acid. Eur Food Res Technol 212: 334-343.

Reshma MV, Saritha SS, Balachandran C, Arumughan C. 2008. Lípase catalyzed interesterification of plam setearin and rice bran oil blends for preparation of zero trans shortening with bioactive phytochemicals. Bioresource Technol 99: 5011-5019.

Rodríguez A, Castro E, Salinas MC, Lpez R, Miranda M. 2001. Interesterification of tallow and sunflower oil. J Am Oil Chem Soc 78: 431-436.

Segura N, da Silva RC, Schafer de M, Soares FA, Gioielli LA, Jachmanián I. 2011a. Valorization of beef tallow by lipasecatalyzed interesterifcation with high oleic sunfower oil. J Am Oil Chem Soc 88: 1945-1954.

Timms RE. 2003. Confectionary fats handbook. Bridgwater, Inglaterra: The Oily Press Eds.

Van Hoed V, Depaemelaere G, Ayala VJ, Santiwattana P, Verhé R, De Greyt W. 2006. Influence of chemical refining on the major and minor components of rice bran oil. J Am Oil Chem Soc 63:315-320.

Walker RE, ed. 1990. Official methods and recommended practices of the American Oil Chemists Society. 4th ed. Champaign, IL: American Oil Chemists Society.

Xu X, Godber JS. 1999. Purification and identification of components of $\gamma$-Oryzanol in rice bran oil. J Agric Food Chem 47: 2724-2728.

Xu X, Skands ARH, Hoy CE, Mu H, Balchen S, Adler-Nissen J. 1998. Production of specific-structured lipids by enzymatic interesterification: Elucidation of acyl migration by response surface design. J Am Oil Chem Soc 75: 1179-1186.

Cite this article as: Segura N, Jachmanián I. 2020. Zero-trans fats by enzymatic interesterification of blends beef tallow / rice bran oil. OCL 27: 4. 